\title{
Traveling pulse on a periodic background in parametrically driven systems
}

\author{
Alejandro O. León* and Marcel G. Clerc ${ }^{\dagger}$ \\ Departamento de Física, Facultad de Ciencias Físicas y Matemáticas, Universidad de Chile, Casilla 487-3, Santiago, Chile \\ Saliya Coulibaly \\ Laboratoire de Physique des Lasers, Atomes et Molécules, CNRS UMR 8523, \\ Université des Sciences et Technologies de Lille-59655 Villeneuve d'Ascq Cedex, France, EU \\ (Received 11 February 2015; published 8 May 2015)
}

\begin{abstract}
Macroscopic systems with dissipation and time-modulated injection of energy, parametrically driven systems, can self-organize into localized states and/or patterns. We investigate a pulse that travels over a one-dimensional pattern in parametrically driven systems. Based on a minimal prototype model, we show that the pulses emerge through a subcritical Andronov-Hopf bifurcation of the underlying pattern. We describe a simple physical system, a magnetic wire forced with a transverse oscillatory magnetic field, which displays these traveling pulses.
\end{abstract}

DOI: 10.1103/PhysRevE.91.050901

PACS number(s): 89.75.Kd, 05.45.Yv, 75.78.-n

Physical systems kept out of thermodynamic equilibrium exhibit pattern formation [1]. This morphogenesis, or transition from a uniform state to a pattern when a control parameter is varied, is understood in terms of a spontaneous symmetry breaking instability of the uniform state (see [1,2], and references therein). Far from the aforementioned transition, the stationary patterns can become unstable, which typically induces rich dynamical behaviors. For example, Andronov-Hopf instabilities originate vacillating-breathing states [3], while the stationary parity-breaking bifurcation induces global [4] or localized drift patterns [5]. These dynamical behaviors can coexist in a large range of parameters [3,6,7]. From a theoretical point of view, Coullet and Iooss [8] classified the generic instabilities of static periodic patterns in one spatial dimension, and described them with amplitude equations for the critical modes. This theory was generalized $[9,10]$ to explain experimental observations, such as topological defects and localized drifting domains.

Let us consider the specific case of parametrically driven systems, that is, systems in which the injection of energy or momentum is time modulated [11]. For a forcing frequency close to twice their natural frequency, parametric systems are known to show a subharmonic resonance [11]. This instability in extended systems is characterized by the formation of subharmonic spatially periodic patterns, such as the wellknown Faraday waves of vibrated fluids [12]. At the onset of subharmonic resonance, the dynamical evolution of the amplitude of oscillations can be described by the parametrically driven, damped nonlinear Schrödinger equation (PDNLS). This prototype model has been used to study selforganization in several physical systems, such as a vertically oscillating layer of water [13,14], ferromagnetic media driven by an oscillatory magnetic field [15-17], parametrically driven nonlinear oscillators [18], localized structures in nonlinear lattices [19], light pulses in optical fibers [20], optical parametric oscillators [21], and spintronic devices [22], to mention a few. Despite the success of the prototype PDNLS

\footnotetext{
*aoleon@dfi.uchile.cl

†marcel@dfi.uchile.cl

${ }_{\ddagger}^{\ddagger}$ saliya.coulibaly@univ-lille1.fr
}

model, it fails to predict localized states with damped spatial oscillations of parametrically forced ferromagnets and coupled oscillators $[17,18]$. To recover the dynamical properties of the original system, the PDNLS equation must be amended by taking into account higher order terms.

The instabilities of patterns in parametrically forced systems, as well as the dissipative structures and spatiotemporal behaviors induced by these bifurcations, are not entirely well understood. In the context of vertically driven granular media, the emergence of secondary drift instability of standing waves has been reported [23]. In the case of a rectangular water container subjected to vertical vibrations, preliminary observations show for forcing amplitudes above a critical value, the amplitude of Faraday waves becomes modulated by a nonpropagative localized structure [cf. Fig. 1(a)] [24]. Traveling pulses on stationary periodic structures have been observed in directional solidification [25]. Theoretically, this type of dynamical behavior has been described in a unified manner by considering the coupling of counterpropagative wave envelopes [10].

The aim of this Rapid Communication is to theoretically and numerically investigate traveling pulses immersed in a one-dimensional pattern in parametrically driven systems. The pulses over patterns are characterized by a localized increment of the amplitude of the spatially periodic background. Figures 1(b) and 1(c) show this type of solution for a parametrically driven ferromagnetic wire. Using an adequate amplitude equation, we show that pulses appear as results of a subcritical Andronov-Hopf bifurcation of the underlying pattern. This minimal approach allows us to predict this dynamical behavior in different physical systems. We show that a magnetic wire forced with a transverse oscillatory magnetic field, displays these traveling pulses.

Unified description of traveling pulses. Let us describe the envelope of the oscillations of a parametric system by a complex order parameter, $A(t, z)$, that obeys the following amended parametrically driven damped nonlinear Schrödinger equation (APDNLS),

$$
\partial_{t} A=-i\left(v A+|A|^{2} A+\partial_{z z} A\right)-\mu A+\gamma \bar{A}+i \delta \bar{A}\left(\partial_{z} A\right)^{2},
$$


(a)

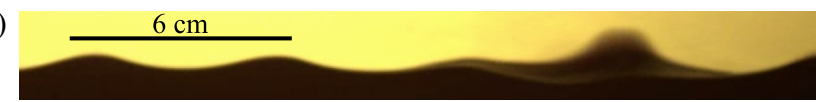

(b)

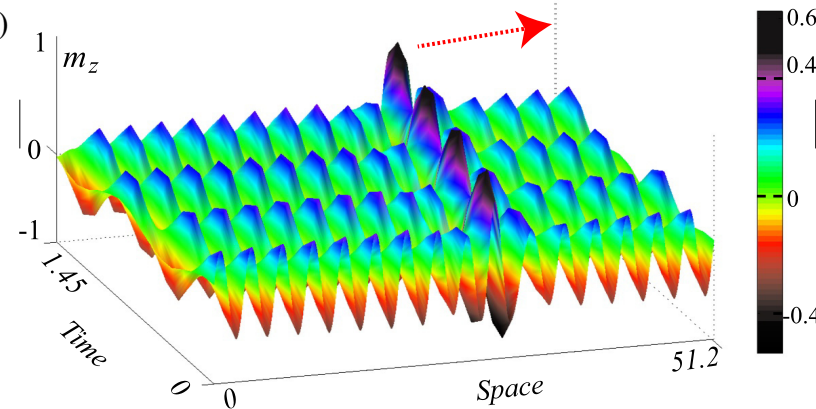

(c)

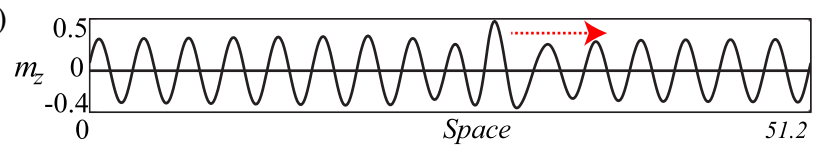

FIG. 1. (Color online) Pulses over a periodic background or pattern in parametrically driven systems. (a) Experimental snapshot of a nonpropagative hydrodynamic pulse supported by Faraday waves [24]. Numerical simulation of a magnetic wire forced with a transverse oscillatory magnetic field, Eq. (2), with $H_{0}=h_{0}=2$, $\beta=4.8, \alpha=0.02$, and $v=4$. (b) Spatiotemporal diagram of a slowly traveling magnetic pulse and (c) instantaneous profile of the magnetic pulse at time $t=0$.

where $t$ and $z$ are the temporal and spatial coordinates. The coefficient $v$ accounts for the detuning between half of the forcing frequency and the response frequency, while the parameters $\mu$ and $\gamma$ account for the dissipation and the parametric injection, respectively. For $\delta=0$, Eq. (1) is the usual PDNLS model, used to describe the parametrically driven systems for small injection and dissipation of energyquasireversible limit [26]. Hence, parameters and operators of the above equation scale as $v \sim \mu \sim \gamma \sim|A|^{2} \sim \partial_{z z} \sim \partial_{t}$. The term proportional to $\delta$ in this limit is a higher order correction. Notice that the instabilities of the quiescent state $A=0$ are not modified by the amending term $i \delta \bar{A}\left(\partial_{z} A\right)^{2}$, because it is nonlinear.

Numerical simulations of the APDNLS equation (1) exhibit spatially periodic patterns for a given forcing amplitude and positive detuning. When $\gamma$ is increased, the model displays traveling pulses over the pattern state. Figure 2 illustrates a single left-traveling pulse, and multiple pulses. Due to the $x \rightarrow$ $-x$ invariance, the same solutions with a right-traveling pulse exist. All numerical simulations of the above equation have been performed with a space discretization of finite differences centered schemes of sixth order, and a fifth order controlled step size Runge-Kutta scheme [27] for temporal evolution.

Multiple pulses can move in the same direction or counterpropagate. In the latter case, the result of the collisions depends on the parameters: the first possibility is that one of the pulses is destroyed after the collision, while in the second case they reemerge from collisions with their particlelike characteristics unchanged [see Fig. 2(c)]. The speed of traveling pulses decreases with the amplitude of the forcing $\gamma$ following a square-root power law (see Fig. 3). For small injections of energy $\gamma<\gamma_{c}$, traveling pulses are not stable, and the pulselike
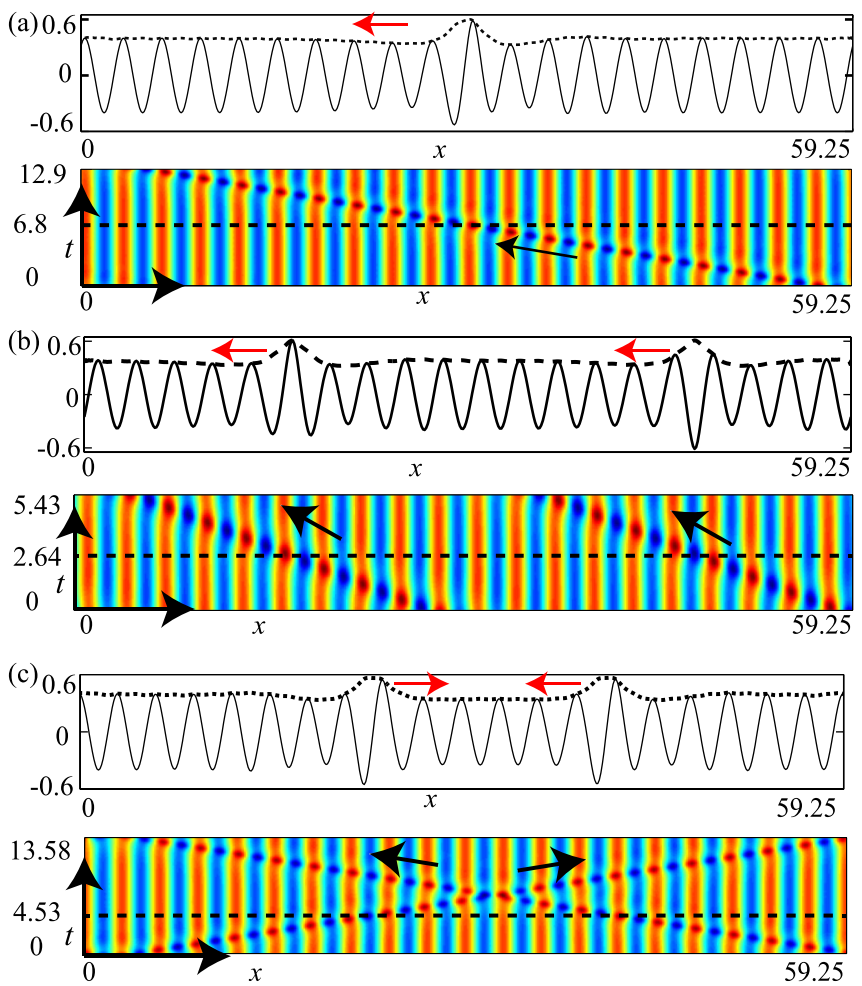

FIG. 2. (Color online) Pulse solutions obtained with different initial conditions. The top profiles of the variable $\operatorname{Re}(A)$ are taken from the dashed line of the respective spatiotemporal diagram. (a) Solitary left-traveling pulses over spatially periodic patterns. (b) Two left-traveling pulses. (c) Collision of two counterpropagative pulses. Parameters are $\gamma=0.5, \mu=0.4, v=4.5$, and $\delta=2$. The simulations use $N=500$ points with a spatial step size $d x=0.1185$.

initial conditions decay to a pattern with a characteristic time $\Delta t \sim\left(\gamma_{c}-\gamma\right)^{-1 / 2}$ (cf. inset of Fig. 3). Then, these two features allow us to conjecture that the traveling pulses appear through a saddle-node bifurcation mechanism. Likewise, increasing the parameter $\gamma>\gamma_{c}$, the pattern that supports the pulses becomes unstable for $\gamma \geq \Gamma_{c}$, and multiple pulselike structures emerge spontaneously and invade the system.

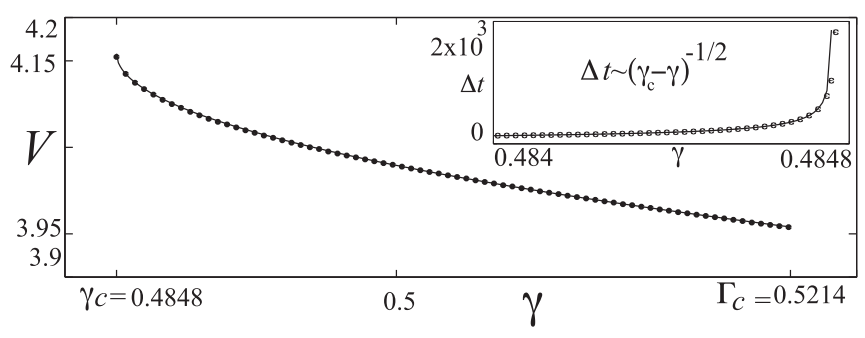

FIG. 3. Speed of the traveling pulses as a function of forcing parameter $\gamma$. Points are obtained from numerical simulations of Eq. (1) with $v=4.5, \delta=2.0$, and $\mu=0.4$. The solid line $V(\gamma)=$ $\left[-a_{2}-\sqrt{a_{2}^{2}-4 a_{1}\left(a_{3}-\gamma\right)}\right] /\left(2 a_{1}\right)$ fits the speed of the pulse with $a_{1}=0.9124, a_{2}=-7.588$, and $a_{3}=16.26$. The inset shows the typical time in which a pulse decays into a pattern state for $\gamma<\gamma_{c}$. The solid curve $\Delta t(\gamma)=2 b_{1} /\left[-b_{2}-\sqrt{b_{2}^{2}-4 b_{1}\left(b_{3}-\gamma\right)}\right]$ fits the decaying times with $b_{1}=-14.227, b_{2}=0.0076504$, and $b_{3}=0.48476$. 
(a)

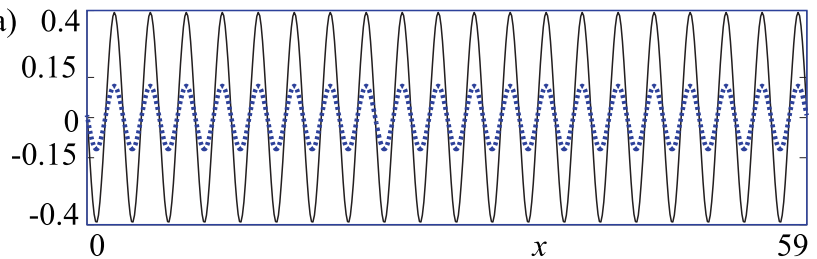

(b)

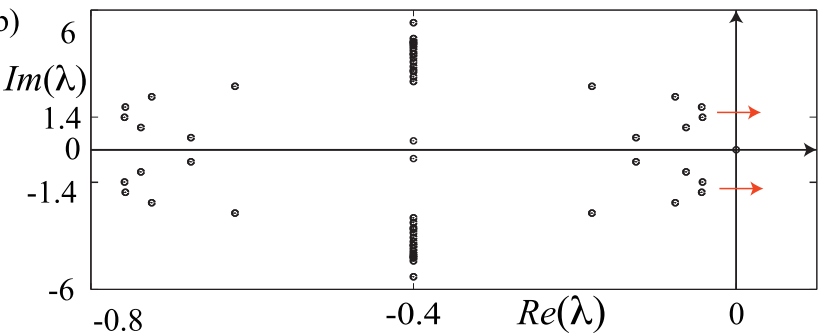

FIG. 4. (Color online) Andronov-Hopf bifurcation of patterns state of amplitude equation (1). (a) Stationary pattern for $\gamma=0.48$, $\mu=0.4, v=4.5, \delta=2$, and $d x=0.1185$. The real and imaginary parts of the amplitude $A(x, t)$ are represented by a continuous and dashed curve, respectively. (b) Eigenvalues of the pattern represented in the complex plane.

The existence, stability properties, and dynamical evolution of traveling pulses immersed in patterns is closely related to the amending term of Eq. (1). Indeed, in the limit $\delta \rightarrow 0$, the APDNLS model, Eq. (1), only exhibits stationary spatially periodic patterns for positive detuning and small amplitude forcing $\gamma$, and vacillating-breathing patterns for higher values of $\gamma$. Hence, traveling pulses have not been observed in the limit of the parametrically driven nonlinear Schrödinger equation. It is worth noting that in the region where patterns exist, the gradients on the amplitude $\left|\partial_{z} A\right| \sim q|A|$ are not small (where $q$ is the pattern wave number), and then the terms like $i \bar{A}\left(\partial_{z} A\right)^{2}, i|A|^{2} \partial_{z z} A, i A^{2} \partial_{z z} \bar{A}$, and $i A\left|\partial_{z} A\right|^{2}$ become relevant in the dynamics of Eq. (1). However, we have observed pulse solutions only when the amending term $i \bar{A}\left(\partial_{z} A\right)^{2}$ is included.

Formation mechanism of traveling pulses. At leading order, the pattern that supports the pulses reads $A \approx T(t, z) e^{i q z}+$ c.c., where $q=\sqrt{v}$ and the slowly varying amplitude of the pattern satisfies $\partial_{t} T=(\gamma-\mu) T-(\delta v-3)^{2} T|T|^{4} /(2 \mu)+$ $(2 \nu / \mu) \partial_{z z} T$. Notice that for $\delta \rightarrow 0$ we recover the amplitude equation of patterns in parametric systems [26,28]. One of the effects of the nonlinear gradient term in the APDNLS equation (1) is to introduce a critical point at $v \equiv 3 / \delta$ for which the quintic order saturation vanishes, changing the type of bifurcation. The dynamics of $T(t, z)$ is of relaxation type, that is, the amplitude evolves minimizing a functional. Consequently, permanent behaviors such as oscillatory patterns and traveling pulses are prohibited, and it is necessary to use the full model, Eq. (1), to understand the formation of traveling pulses.

The analytical stability analysis of the spatially periodic pattern states from Eq. (1) is a tricky task. However, its numerical study is easily accessible. Figure 4 shows the typical stationary pattern solution and its corresponding eigenvalues. When the $\gamma$ parameter is increased, two complex conjugate eigenvalues cross the imaginary axis, as illustrated in Fig. 4(b). This instability corresponds to an Andronov-Hopf bifurcation. Hence, the critical modes of this instability are of the type

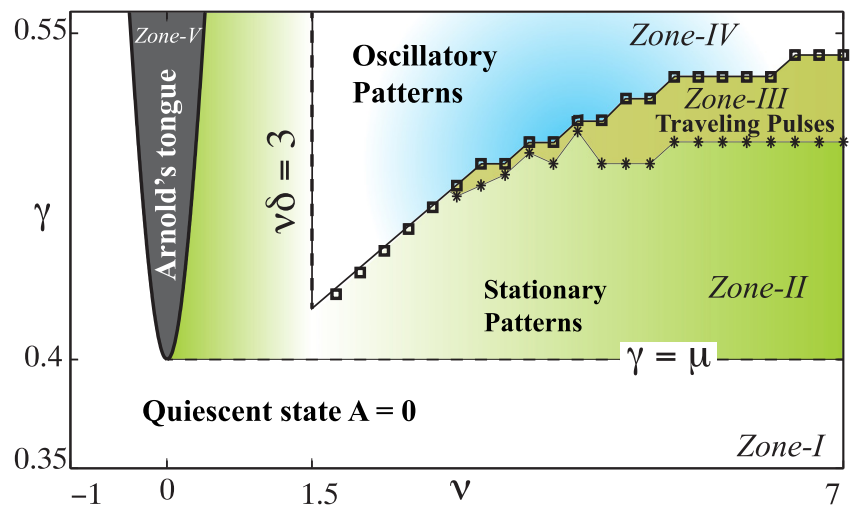

FIG. 5. (Color online) Bifurcation diagram of APDNLS equation (1). In zone I the state $A=0$ is the only equilibrium. Zone $\mathrm{V}$ is Arnold's tongue; inside this region the trivial state $A=0$ is unstable. The pattern states are observed in zones II, III, and V. The squares denote the transition from stationary patterns to oscillatory patterns. Traveling pulses are observed in zone III.

of left- and right-traveling waves, which can be written in the form $A_{c, \pm}(t, z)=B_{ \pm}(T, Z) e^{i(\omega t \pm k z)} f_{q}( \pm z)$, where $k$ is a real constant, $f_{q}$ is a complex-valued $q$-periodic function, and $B_{ \pm}(T, Z)$ is a slowly varying envelope in time $T$ and space $Z$. When the spatially periodic pattern $\left[B_{ \pm}(T, Z)=0\right]$ is unstable, multiple pulselike structures emerge spontaneously and invade the system, which is equivalent to having an inhomogeneous profile in $B_{ \pm}(T, Z)$. Uniform profiles in $B_{ \pm}(T, Z)$ are not numerically observed. It is important to note that this paritybreaking discontinuous or subcriticalsecondary bifurcation is numerically found for $v>3 / \delta$. Figure 5 shows the phase diagram of APDNLS equation (1). In zone I only the quiescent state $A=0$ is observed for positive detuning. If one increases the forcing parameter $\gamma$, the system exhibits the emergence of patterns, zone II, through a supercritical bifurcation at $\gamma=\mu$. These spatially periodic patterns correspond to subharmonic waves in original parametrically driven systems. The pattern states are observed in zones II, III and V. Furthermore, for sufficiently large values of $\gamma$ and $v$, patterns become oscillatory, zone IV, through a subcritical Andronov-Hopf bifurcation. In this zone the system exhibits traveling waves. As a result of the subcritical Andronov-Hopf bifurcation, traveling pulses are observed in the region of coexistence between stationary and oscillatory patterns, zone III. Decreasing $\delta$, both regions of oscillatory patterns with broken $x \rightarrow-x$ symmetry, and traveling pulses move to larger detuning. Therefore, in quasireversible limit, $\delta=0$, those areas vanish from the bifurcation diagram.

Parametrically driven magnetic wire. Let us consider a ferromagnetic wire along the $z$ axis. In the continuous framework, the material is described by its normalized magnetization $\mathbf{m}=$ $\mathbf{m}(t, z)$, where $\{z, t\}$ are the spatial coordinates along the wire and the time, respectively. The evolution of the magnetization obeys the Landau-Lifshitz-Gilbert equation [29],

$$
\frac{\partial \mathbf{m}}{\partial t}=-\mathbf{m} \times \mathbf{h}_{\mathrm{eff}}+\alpha \mathbf{m} \times \frac{\partial \mathbf{m}}{\partial t} .
$$

The first term on the right-hand side of Eq. (2) accounts for the conservative precessions generated by the effective field, 
$\mathbf{h}_{\text {eff }} \equiv h \mathbf{e}_{x}-\beta m_{z} \mathbf{e}_{z}+\partial_{z z} \mathbf{m}$, where $\left\{\mathbf{e}_{x}, \mathbf{e}_{y}, \mathbf{e}_{z}\right\}$ denote the unit vectors along the respective Cartesian axis and $h$ is the intensity of the external field $\mathbf{h}=h \mathbf{e}_{x}$. The coefficient $\beta>0$ accounts for the anisotropy of the wire and it penalizes the magnetization along the $z$ axis. Finally, the Laplacian of the magnetization stands for the ferromagnetic exchange interaction and it favors the homogeneous magnetic configurations.

The second term of Eq. (2) is the phenomenological Gilbert damping. Equation (2) has two trivial equilibria, $\mathbf{m}= \pm \mathbf{e}_{x}$. For positive external field, $h>0, \mathbf{m}=\mathbf{e}_{x}$ is the most favored state. The dynamics around this equilibrium is characterized by damped oscillations with frequency $\omega_{0}=\sqrt{H_{0}\left(H_{0}+\beta\right)}$. Considering a combination of a constant and a periodic external magnetic field, $h(t)=H_{0}+h_{0} \cos \left[2\left(\omega_{0}+v\right) t\right]$, the magnetic wire behaves as a parametrically driven oscillatory medium. Therefore, in a certain range of parameters the system must exhibit traveling pulse immersed in patterns. Figure 1 shows the profile and spatiotemporal evolution of traveling pulses observed in the magnetic wire.

To understand the origin of these pulses, we consider the following ansatz:

$$
\begin{aligned}
\left(\begin{array}{l}
m_{y} \\
m_{z}
\end{array}\right)= & \sqrt{\frac{4 \omega_{0}}{\beta\left(\omega_{0}^{2}+3 H_{0}^{2}\right)}} A(t, z) e^{i \phi(t)}\left(\begin{array}{c}
\sqrt{H_{0}+\beta} \\
-i \sqrt{H_{0}}
\end{array}\right) \\
& + \text { c.c. }+\vec{W},
\end{aligned}
$$

with $m_{x}=\sqrt{1-m_{y}^{2}-m_{z}^{2}}, \phi(t)=\omega_{0} t+v t+\pi / 4$, the symbol c.c. stands for complex conjugate, and $\vec{W}$ is a small correction vector that accounts for the higher order terms in the amplitude A. Replacing the above ansatz in Eq. (2), linearizing in $\vec{W}$, and imposing a solvability condition, after straightforward calculations, we obtain

$$
\begin{aligned}
\partial_{t} A= & -i\left(v A+|A|^{2} A+\partial_{\xi \xi} A\right)-\mu A+\gamma \bar{A}+i c_{1}|A|^{2} \partial_{\xi \xi} A \\
& +i c_{2} A^{2} \partial_{\xi \xi} \bar{A}+i c_{3} A\left|\partial_{\xi} A\right|^{2}+i c_{1} \bar{A}\left(\partial_{\xi} A\right)^{2}
\end{aligned}
$$

where $\quad \mu \equiv \alpha\left(2 H_{0}+\beta\right) / 2, \quad \gamma \equiv \beta h_{0} /\left(4 \omega_{0}\right), \quad c_{1}=$ $d \beta\left(4 H_{0}-\beta\right) / 2, \quad c_{2}=-d\left(\beta^{2}+12 H_{0} \beta+16 H_{0}^{2}\right) / 4$, $c_{3}=-d\left(\beta^{2}+4 H_{0} \beta+8 H_{0}^{2}\right), \quad d=4 \omega_{0} /\left[\beta H_{0}\left(2 H_{0}+\right.\right.$ $\left.\beta)\left(4 H_{0}+\beta\right)\right]$, and the spatial coordinate $\xi$ is related to the original coordinate by $\xi \equiv \sqrt{2 \omega_{0} /\left(2 H_{0}+\beta\right)} z$. In the quasireversible limit, the last four terms in the above equation are negligible. In this limit the forcing magnetic wire is described by the PDNLS equation. This equation allows one to study different localized states such as dissipative solitons [15,30-35] and localized waves [17,36]. Both Eqs. (1) and (4) share the same pattern instability and traveling pulses. Hence, the magnetic wire forced with a transverse oscillatory magnetic field can be modeled phenomenologically by the amplitude equation (1).

In conclusion, we have studied the emergence of traveling pulses immersed in one-dimensional patterns in the context of parametric systems. As results of the subcritical parity-breaking Andronov-Hopf bifurcation of the stationary pattern, the system exhibits a coexistence region between stable stationary and unstable oscillatory patterns. Within this coexistence region, we observe traveling pulses. Depending on the initial condition, we found a single and multiple pulses solutions. A simple PDNLS-like model allows us to explain the pulses dynamics as an effect of the nonlinear gradients of the full system. This simple model also allows us to predict traveling pulses in a magnetic wire forced with an external transverse oscillatory magnetic field.

Acknowledgments. A.O.L. thanks U. Pereira for fruitful discussions and gratefully acknowledges financial support from Becas Conicyt 2012, Contract No. 21120878. M.G.C. acknowledges the financial support of FONDECYT Project No. 1150507. S.C. acknowledges the financial support of Ministry of Higher Education and Research, Nord-Pas de Calais Regional Council and ERDF through the CPER 20072013, as well as by the ANR LABEX CEMPI project (ANR11-LABX-0007). The preliminary experimental image of the nonpropagative hydrodynamic pulse [Fig. 1(a)] is courtesy of Dr. Ignacio Espinoza B.
[1] G. Nicolis and I. Prigogine, Self-Organization in Nonequilibrium Systems (Wiley, New York, 1977).

[2] M. C. Cross and P. C. Hohenberg, Pattern formation outside of equilibrium, Rev. Mod. Phys. 65, 851 (1993).

[3] P. Brunet, J. M. Flesselles, and L. Limat, Elastic properties of a cellular dissipative structure, Euro. Phys. J. B 35, 525 (2003).

[4] L. Pan, and J. R. de Bruyn, Spatially uniform traveling cellular patterns at a driven interface, Phys. Rev. E 49, 483 (1994).

[5] P. Coullet, R. E. Golstein, and G. H. Gunaratne, Parity-Breaking Transitions of Modulated Patterns in Hydrodynamic Systems, Phys. Rev. Lett. 63, 1954 (1989).

[6] C. Counillon, L. Daudet, T. Podgorski, and L. Limat, Dynamics of a Liquid Column Array under Periodic Boundary Conditions, Phys. Rev. Lett. 80, 2117 (1998).

[7] P. Brunet, Stabilized Kuramoto-Sivashinsky equation: A useful model for secondary instabilities and related dynamics of experimental one-dimensional cellular flows, Phys. Rev. E 76, 017204 (2007).
[8] P. Coullet and G. Iooss, Instabilitiy of One-Dimensional Cellular Patterns, Phys. Rev. Lett. 64, 866 (1990).

[9] L. Gil, Instabilities of one-dimensional cellular patterns: Far from the secondary threshold, Europhys. Lett. 48, 156 (1999).

[10] L. Gil, Secondary instability of one-dimensional cellular patterns: A gap soliton, black soliton and breather analogy, Physica D 147, 300 (2000).

[11] L. D. Landau and E. M. Lifshiftz, Mechanics, Course of Theoretical Physics Vol. 1 (Pergamon, New York, 1976).

[12] M. Faraday, On a peculiar class of acoustical figures; and on certain forms assumed by groups of particles upon vibrating elastic surfaces, Philos. Trans. R. Soc. London 121, 299 (1831).

[13] J. W. Miles, Parametrically excited solitary waves, J. Fluid Mech. 148, 451 (1984).

[14] M. G. Clerc, S. Coulibaly, N. Mujica, R. Navarro, and T. Sauma, Soliton pair interaction law in parametrically driven Newtonian fluid, Philos. Trans. R. Soc., A 367, 3213 (2009).

[15] I. V. Barashenkov, M. M. Bogdan, and V. I. Korobov, Stability diagram of the phase-locked solitons in the parametrically 
driven, damped nonlinear Schrödinger equation, Europhys. Lett. 15, 113 (1991).

[16] S. R. Woodford and I. V. Barashenkov, Stability of the Bloch wall via the Bogomolnyi decomposition in elliptic coordinates, J. Phys. A: Math. Theor. 41, 185203 (2008).

[17] M. G. Clerc, S. Coulibaly, and D. Laroze, Localized states of parametrically driven easy-plane ferromagnetic wire, Physica D 239, 72 (2010).

[18] M. G. Clerc, S. Coulibaly, and D. Laroze, Localized states beyond the asymptotic parametrically driven amplitude equation, Phys. Rev. E 77, 056209 (2008).

[19] B. Denardo, B. Galvin, A. Greenfield, A. Larraza, S. Putterman, and W. Wright, Observations of localized structures in nonlinear lattices: Domain walls and kinks, Phys. Rev. Lett. 68, 1730 (1992).

[20] J. N. Kutz, W. L. Kath, R.-D. Li, and P. Kumar, Longdistance pulse propagation in nonlinear optical fibers by using periodically spaced parametric amplifiers, Opt. Lett. 18, 802 (1993).

[21] S. Longhi, Stable multipulse states in a nonlinear dispersive cavity with parametric gain, Phys. Rev. E 53, 5520 (1996).

[22] A. O. León and M. G. Clerc, Spin-transfer-driven nanooscillators are equivalent to parametric resonators, Phys. Rev. B 91, 014411 (2015).

[23] S. Douady, S. Fauve, and C. Laroche, Subharmonic instabilities and defects in a granular layer under vertical vibrations, Europhys. Lett. 8, 621 (1989).

[24] I. Espinoza, Control de solitones disipativos en un fluido forzado paramétricamente, Pontificia Universidad Católica de Chile, 2009.

[25] A. J. Simon, J. Bechhoefer, and A. Libchaber, Solitary Modes and the Eckaus Instability in Directional Solidification, Phys. Rev. Lett. 61, 2574 (1988).
[26] M. G. Clerc, S. Coulibaly, and D. Laroze, Parametrically driven instabilities in quasi-reversal systems, Int. J. Bifurcation Chaos 19, 3525 (2009).

[27] W. H. Press, S. A. Teukolsky, W. T. Vetterling, and B. P. Flannery, Numerical Recipes in C: The Art of Scientific Computing (Cambridge University Press, New York, 1992).

[28] P. Coullet, T. Frisch, and G. Sonnino, Dispersion-induced patterns, Phys. Rev. E 49, 2087 (1994).

[29] I. D. Mayergoyz, G. Bertotti, and C. Serpico, Nonlinear Magnetization Dynamics in Nanosystems (Elsevier, Oxford, 2009).

[30] I. V. Barashenkov and E. V. Zemlyanaya, Traveling solitons in the damped-driven nonlinear Schrödinger equation, SIAM J. Appl. Math. 64, 800 (2004).

[31] I. V. Barashenkov, E. V. Zemlyanaya, and T. C. van Heerden, Time-periodic solitons in a damped-driven nonlinear Schrödinger equation, Phys. Rev. E 83, 056609 (2011).

[32] I. V. Barashenkov and E. V. Zemlyanaya, Soliton complexity in the damped-driven nonlinear Schrödinger equation: Stationary to periodic to quasiperiodic complexes, Phys. Rev. E 83, 056610 (2011).

[33] D. Urzagasti, D. Laroze, M. G. Clerc, S. Coulibaly, and H. Pleiner, Two-soliton precession state in a parametrically driven magnetic wire, J. Appl. Phys. 111, 07D111 (2012).

[34] M. G. Clerc, M. A. Garcia-Ñustes, Y. Zárate, and S. Coulibaly, Phase shielding soliton in parametrically driven systems, Phys. Rev. E 87, 052915 (2013).

[35] D. Urzagasti, D. Laroze, M. G. Clerc, H. Pleiner, Breather soliton solutions in a parametrically driven magnetic wire, Europhys. Lett. 104, 40001 (2013).

[36] M. G. Clerc, S. Coulibaly, and D. Laroze, Localized waves in a parametrically driven magnetic nanowire, Europhys. Lett. 97, 30006 (2012). 